\title{
L'influence de la conceptualisation métaphorique sur les choix traductionnels
}

L'exemple de l'astrophysique et des trous noirs

Charlène Meyers

\section{CpenEdition \\ Journals}

\section{Édition électronique}

URL : http://journals.openedition.org/asp/4583

DOI : 10.4000/asp.4583

ISSN : 2108-6354

\section{Éditeur}

Groupe d'étude et de recherche en anglais de spécialité

\section{Édition imprimée}

Date de publication : 1 novembre 2014

Pagination : 83-101

ISSN : 1246-8185

Référence électronique

Charlène Meyers, "L'influence de la conceptualisation métaphorique sur les choix traductionnels », ASp [En ligne], 66 | 2014, mis en ligne le 01 novembre 2015, consulté le 02 novembre 2020. URL: http://journals.openedition.org/asp/4583 ; DOI : https://doi.org/10.4000/asp.4583

Ce document a été généré automatiquement le 2 novembre 2020.

Tous droits réservés 


\title{
L'influence de la conceptualisation métaphorique sur les choix traductionnels
}

\author{
L'exemple de l'astrophysique et des trous noirs
}

\section{Charlène Meyers}

\section{Introduction}

1 Dans cet article, nous avons voulu tester l'efficacité de quelques outils sollicités par les étudiants-traducteurs pour repérer les métaphores conceptuelles, fréquentes chez les chercheurs qui étudient les trous noirs, et pour les traduire. Dans un premier temps, nous montrons pourquoi certaines bases de données terminologiques, certains thésaurus, lexiques ou glossaires ne permettent pas à l'étudiant-traducteur de tirer les meilleures conclusions en ce qui concerne la traduction du concept de «black hole». Ensuite, nous expliquons pourquoi une analyse qualitative à partir de quelques textes semble constituer la meilleure solution parmi celles qui s'offrent au traducteur pour repérer les métaphores conceptuelles et pour traduire les expressions métaphoriques qui en découlent. À l'aide d'exemples extraits de deux corpus comparables, nous analysons successivement la manière dont la notion de trou noir est conceptualisée en anglais et en français afin de vérifier si celle-ci est gouvernée par les mêmes métaphores dans les deux langues et si les expressions métaphoriques qui en découlent sont sensiblement les mêmes. Enfin, à partir de cette analyse, nous déduisons la traduction qui semble la plus adéquate pour le concept de «black hole» et montrons pourquoi certaines recommandations des bases de données semblent entrer en contradiction avec la métaphore conceptuelle du concept de trou noir.

2 La place de la métaphore en science fait débat depuis de nombreuses années. Dans les années 1930, Eugen Wüster, ingénieur et fondateur de la terminologie, plaidait en faveur d'une normalisation de la langue spécialisée afin de garantir la monosémie et la monoréférentialité de chaque terme. C'est ce que nous explique Hildegund Bühler : 
Wüster's lifelong concern was with questions of terminology or rather special language terminology and its standardization. (Bühler $1982: 425$ ) socioterminologie, ils ont remis en question la théorie wüstérienne, soulignant l'importance des métaphores pour constituer de nouveaux termes :

Les métaphores et l'histoire dans laquelle elles s'inscrivent constituent deux aspects des terminologies qui mettent en question les lieux communs concernant la monosémie des termes et la validité d'une partition du savoir en domaines. (Gaudin $2003: 205$ )

Instead of promoting the curtailing of metaphorical language in scientific discourse, as the principles of traditional Terminology suggest, one might even consider encouraging metaphor for the sake of progress in understanding. More research on the process of metaphorical understanding and its relevance to language is needed. (Temmerman $2000: 156)$

En outre, dans leur ouvrage intitulé Les Métaphores dans la vie quotidienne (1986 [1980]), George Lakoff et Mark Johnson ont réfuté l'hypothèse selon laquelle les métaphores n'étaient que des ornements discursifs. Ils ont au contraire défendu l'idée qu'elles constituent un système universel et fréquent de conceptualisation.

6 Les métaphores n'intéressent pas seulement les linguistes. Les scientifiques se sont également penchés sur la question. Dans le premier chapitre de son ouvrage Making truth: the role of metaphor in science (2003), Theodore Brown, professeur émérite de chimie de l'Université d'Illinois, relève le caractère omniprésent des métaphores en science :

This book is concerned with an aspect of science that is largely absent from textbooks yet is central to an understanding of the nature of science. I want to show that much of what scientists do - how they conceive of productive experiments, what they observe, and their interpretations of observations - is governed by metaphorical reasoning. (Brown $2003: 2$ )

George Lakoff et Rafael Núñez, spécialistes en sciences et linguistique cognitives, ont de leur côté examiné le monde des mathématiques :

We will argue, in our discussion [...] that conceptual metaphor is the central cognitive mechanism of extension from basic arithmetic to such sophisticated applications of number. Moreover, we will argue that a sophisticated understanding of arithmetic itself requires conceptual metaphors using nonnumerical mathematical source domains. (Lakoff \& Núñez $2000: 47)$

Dans son ouvrage intitulé Models and Analogies in Science (1966), Mary B. Hesse s'est également intéressée au rôle des métaphores, mais cette fois dans le domaine de la philosophie des sciences.

Mentionnons enfin le philosophe Richard Boyd qui a forgé le concept de «métaphore constitutive d'une théorie »en tentant d'expliquer le rôle de différents types de métaphores dans le développement des disciplines scientifiques :

[...] there are metaphors which scientists use in expressing theoretical claims for which no adequate paraphrase is known. Such metaphors are constitutive of the theories they express, rather than merely exegetical. (Boyd 1993 [1979] : 486) 
$10 \mathrm{Au}$ vu de ce qui vient d'être énoncé se profile l'idée que les métaphores sont inhérentes à tout domaine scientifique. C'est d'ailleurs ce que soutiennent Sylvie Vandaele et Catherine Resche :

Il n'est pas courant d'envisager la langue de la science comme étant une langue métaphorique. Et pourtant, que seraient les théories scientifiques sans la métaphore? (Vandaele $2006: 282$ )

La métaphore constitutive d'une théorie n'a pas pour fonction première de simplifier la compréhension des non-spécialistes, mais de permettre au chercheur, au scientifique, de conceptualiser une nouvelle théorie, de l'aider à concevoir un nouvel angle d'approche pour un domaine donné. (Resche $2002: 13$ )

11 De notre point de vue, il nous paraît justifié d'adopter le cadre théorique selon lequel les métaphores ne se résument pas à des figures de style, mais constituent un puissant outil de réflexion et d'innovation scientifique. Nous adhérons également à la position de G. Lakoff selon laquelle les métaphores permettent de construire une nouvelle représentation mentale à partir d'une représentation déjà existante et que les métaphores dites conceptuelles sont le fruit de la projection (aussi appelée mapping) d'un cadre conceptuel source sur un cadre conceptuel cible :

In short, the locus of metaphor is [...] in the way we conceptualize one mental domain in terms of another. The general theory of metaphor is given by characterizing such cross-domain mappings. And in the process, everyday abstract concepts like time, states, change, causation, and purpose also turn out to be metaphorical. The result is that metaphor (that is, cross-domain mapping) is absolutely central to ordinary natural language semantics. (Lakoff 1993 [1979] : 203)

12 Conceptualiser revient à faire appel à un savoir connu (faisant partie du domaine source) pour expliquer un savoir inconnu ou un aspect particulier de ce savoir (appartenant au domaine cible). La phrase « LA DISCUSSION, C'EST LA GUERRE » est un des exemples de métaphore conceptuelle énoncés par G. Lakoff et M. Johnson, qui témoigne de la projection du cadre conceptuel de la guerre sur celui de la discussion et qui a donné naissance à des expressions métaphoriques qui y sont directement liées, telles que «il a attaqué chacun de mes arguments », «il a mal défendu sa position» (1986: 14). Il faut distinguer métaphore conceptuelle et expression métaphorique: la métaphore conceptuelle est le mécanisme de projection qui s'opère au cœur de notre pensée, tandis que les expressions métaphoriques constituent les marqueurs linguistiques de cette projection (Vandaele $2006: 282$ ).

13 Dès lors, il semble essentiel que le traducteur puisse repérer et comprendre ces métaphores afin de traduire de façon optimale les expressions métaphoriques d'un texte de n'importe quel domaine. L'accès aux métaphores conceptuelles est d'autant plus important pour le traducteur que les modes de conceptualisation, c'est-à-dire les différentes voies qu'emprunte la pensée pour conceptualiser une notion, peuvent fortement varier d'une langue à une autre, même au sein d'un domaine commun. Il est dès lors primordial que le traducteur repère aussi bien dans la langue source que dans la langue cible ces différents modes de conceptualisation.

Examinons un exemple concret de problème de traduction lié aux métaphores conceptuelles : S. Vandaele (2002) a montré qu'il est impossible de proposer une même traduction de " be involved » dans les phrases "Calcium channels are involved in some heart diseases » et "Calcium channels are involved in neuronal functioning ». Même si ces phrases sont extrêmement similaires, elles sont pourtant gouvernées par des scénarios cognitifs différents. Dans la première phrase, il est question d'un processus pathologique, tandis 
que, dans la deuxième phrase, il est question d'un processus physiologique. Ainsi, en analysant plusieurs exemples témoignant de processus similaires, $\mathrm{S}$. Vandaele a dégagé deux métaphores conceptuelles : LA RECHERCHE DE LA CAUSE D'UNE AFFECTION EST UNE ENQUÊTE CRIMINELLE et LES PROCESSUS PHYSIOPATHOLOGIQUES SONT DES SCÉNARIOS. Par conséquent, dans la première phrase, les canaux calciques sont considérés comme des agents ayant une action néfaste. Dès lors, le recours au verbe "impliquer " pour traduire "be involved " se justifie pleinement, puisque ce verbe signifie que les agents sont liés à une affaire fâcheuse. Dans la deuxième phrase, le verbe « agir » constitue une bonne solution pour traduire "be involved», car dans ce cas-ci, les canaux calciques sont considérés comme des acteurs qui suivent différents scénarios (Vandaele 2002 : 226-232). Cet exemple montre que les métaphores conceptuelles influencent fortement les processus de décision traductionnelle.

\section{Les bases de données terminologiques et autres ressources}

Nous avons constaté que l'étudiant-traducteur a souvent tendance à se tourner en premier lieu vers les bases terminologiques pour résoudre des problèmes ponctuels de traduction en langue de spécialité. Cela est probablement dû au fait que le corps enseignant recommande aux étudiants de se tourner vers ce genre d'outil en traduction spécialisée, ainsi qu'à la place prépondérante que prend le cours de terminologie en fin de cycle. (En effet, dans la présente étude, nous prenons en considération les choix de traduction faits par des étudiants en Master 2 en traduction multidisciplinaire. Dans ce type de Master, les étudiants suivent notamment des cours de traduction scientifique et technique, traduction économique, et traduction juridique). Nous avons cherché à savoir si certaines bases de données et d'autres ressources telles que des glossaires pouvaient fournir un accès aux métaphores conceptuelles qui se manifestent dans les études scientifiques ou les textes de vulgarisation en langue anglaise sur les trous noirs.

Tout d'abord, certaines bases ne proposent aucun équivalent pour le terme «black hole ». C'est le cas de la banque de données terminologique du service de la langue française de la Communauté française de Belgique, de FranceTerme et de la base de données lexicographiques panfrancophone (BDLP).

Ensuite, en lançant une recherche sur Termium Plus, nous constatons que la base de données propose deux équivalents en français pour le terme «black hole» qui sont «trou noir» et «astre occlus». Sur la fiche terminologique, figure également la recommandation suivante:

On dit trou noir, mais ça n'est pas un trou et ce n'est pas noir. Ce terme vient de la traduction américaine. Il est plus juste de dire "astre occlus». Astre parce que stellaire, et occlus parce que refermé sur lui-même, caché à lui-même [...]

18 La lecture de la fiche terminologique donne dès lors à l'étudiant l'information qu'il existe deux traductions pour «black hole », celle de " astre occlus » étant préférable. Un constat similaire est observable dans le Grand Dictionnaire Terminologique qui propose également "astre occlus » et «trou noir » comme termes privilégiés pour traduire " black hole ", mais qui, en revanche, n'en recommande aucun en particulier.

En consultant ces deux bases de données terminologiques, le traducteur aura vraisemblablement tendance à décider de traduire «black hole » par « astre occlus ». En 
outre, il n'aura pas accès aux modes de conceptualisation qui gouvernent cette notion en anglais et en français. Par conséquent, il ne bénéficiera d'aucun indice sur la manière de traduire les expressions métaphoriques rencontrées dans le texte source.

Nous avons également exploré un thésaurus, ainsi que dans un lexique et un glossaire d'astronomie et d'astrophysique. Le lexique de Planète Astronomie ${ }^{1}$ ne propose aucune entrée pour les termes «trous noirs » ou " astre occlus ». Le constat est le même pour le glossaire du site internet de l'Espace des sciences ${ }^{2}$, qui est un centre français de culture scientifique, technique et industrielle. Nous avons également testé MOTBIS, un thésaurus français financé par le ministère de l'Éducation nationale, de l'Enseignement supérieur et de la Recherche ${ }^{3}$. Notre recherche nous a menés à une fiche indiquant que " le terme 'trou noir' est employé pour le terme 'astre occlus' ».

Nous avons tenté de comprendre pourquoi certaines ressources proposent une terminologie multiple pour traduire "black hole ", ou recommandent l'utilisation du terme "astre occlus». Premièrement, nous supposons que certaines ressources proposent une terminologie double ou recommandent l'usage du terme " astre occlus » au détriment de "trou noir" dans un souci de combattre les anglicismes et les traductions littérales et de préserver la langue française. C'est en tout cas l'un des principaux enjeux évoqués dans le plan stratégique en matière de politique linguistique émis par le gouvernement québécois en 2009 et qui reste en vigueur en 2014 :

Le français, langue officielle du Québec, est une valeur fondamentale de la société québécoise. Le Québec, seule province canadienne dans laquelle la majorité de la population est de langue maternelle française, s'est doté depuis plus de trente ans d'une politique linguistique afin de promouvoir l'utilisation du français comme langue commune de la vie publique, dans un environnement nord-américain et une économie mondiale de plus en plus intégrée où domine la langue anglaise. (Boucher, Ouellon \& Gosselin $2009: 7$ )

Il en va de même pour la politique linguistique recommandée par l'Académie française :

Jugeant que la concurrence de l'anglais, même dans la vie courante, représentait une réelle menace pour le français et que les importations anglo-américaines dans notre lexique devenaient trop massives, les autorités gouvernementales ont été amenées, depuis une trentaine d'années, à compléter le dispositif traditionnel de régulation de la langue. À partir de 1972, des commissions ministérielles de terminologie et de néologie sont constituées. Elles s'emploient à indiquer, parfois même à créer, les termes français qu'il convient d'employer pour éviter tel ou tel mot étranger, ou encore pour désigner une nouvelle notion ou un nouvel objet encore innommés. (Académie française non daté)

De plus, nous émettons l'hypothèse que la notion d'astre occlus désigne peut-être une ancienne conceptualisation métaphorique à l'époque où les connaissances sur ce concept n'étaient qu'à leurs balbutiements et qui aurait ensuite subi plusieurs mutations en écho aux nouvelles découvertes concernant les objets considérés. On pourrait donc concevoir chaque grande modification de conceptualisation métaphorique du concept de trou noir comme étant la marque d'un changement de paradigme ou d'une rupture épistémologique dans le domaine de l'astrophysique. C. Resche explique d'ailleurs que l'étude de l'évolution des métaphores dans un domaine précis peut servir d'indicateur de la pensée de scientifique :

Indéniablement, l'observation et l'analyse des métaphores conceptuelles au fil du temps peuvent servir de baromètre de la pensée scientifique. (Resche 2002 : 104)

Dès lors, en observant l'évolution terminologique du concept de trou noir, nous supposons que ce dernier a subi deux grandes mutations qui seraient le reflet des 
avancées dans la connaissance des trous noirs : le concept a tout d'abord pris les noms de "corps invisibles", "corps obscurs" ou encore "d'astre occlus" qui ont été remplacés par l'éponyme « Schwarzschild-Körper », avant que le terme «black hole » ne s'impose.

C'est ce que nous explique une rapide recherche historique. Le marquis de Laplace fut l'un des premiers scientifiques à nommer le concept par les termes "corps invisible " et « corps obscur » qu'il décrit, en 1798, de la manière suivante :

Tous ces corps devenus invisibles, sont à la place où ils ont été observés, puisqu'ils n'en ont point changé, durant leur apparition; il existe dans l'espace céleste, des corps obscurs aussi considérables et peut-être en aussi grand nombre que les étoiles. Un astre lumineux, de même densité que la terre, et dont le diamètre serait 250 fois plus grand que celui du soleil, ne laisserait, en vertu de son attraction, parvenir aucun de ses rayons jusqu'à nous. Il est donc possible que les plus grands corps lumineux de l'univers soient par cela même invisibles. (Laplace 1798: 347-348)

Dans cette définition, Laplace semble décortiquer la métaphore conceptuelle qu'il a créée en appelant ce concept "corps invisible» ou «corps obscur». Il semble que le concept ait aussi pris la désignation "d'astre occlus», que certains astrophysiciens attribuent également à Laplace :

L'idée du trou noir avait déjà fait son apparition avant l'avènement de la Relativité. [...] Le mathématicien français Pierre Simon de Laplace l'avait mentionnée dans son œuvre maîtresse, Le Système du monde. [...] Bien sûr, l'appellation du «trou noir » n'avait pas encore fait son entrée en scène à cette époque, et Laplace appelait ces objets des « astres occlus ». (Xuan Thuan 1998 : 232)

Le terme même de trou noir n'a été proposé qu'en 1968; on parlait auparavant d'astre invisible ou occlus [...] (Luminet $2011: 84$ )

Le concept tombe ensuite dans l'oubli et ne réapparaît qu'au XX $\mathrm{X}^{\mathrm{e}}$ siècle, lorsqu'Albert Einstein pose les principes de la relativité générale qui va profondément modifier la manière d'étudier ces objets célestes. En 1916, l'Allemand Karl Schwarzschild découvre que la théorie de la relativité permettrait d'expliquer l'existence de corps d'une masse effondrée en un point. Le concept prit alors le nom de "Schwarzschild-Körper " par éponymie. Les découvertes de Schwarzschild ont mené à une première mutation du concept, même si l'on ne peut qualifier cette mutation de métaphorique, étant donné que l'éponymie a ici remplacé la métaphore conceptuelle initiale. C'est finalement en 1967 que le physicien John Wheeler de l'Université de Princeton utilisa le terme «black hole » pour désigner le concept, à l'occasion d'un discours (Lombry $2000: 1$ ). Dans un article dédié à ce physicien sur le site de l'Université de Princeton, nous comprenons que le choix de J. Wheeler d'utiliser une métaphore plutôt qu'un terme complexe a fait l'objet d'une démarche consciente :

In the fall of 1967, [John Wheeler] was invited to give a talk on pulsars, thenmysterious deep-space objects, at NASA's Goddard Institute of Space Studies in New York. As he spoke, he argued that something strange might be at the center, what he called a gravitationally completely collapsed object. But such a phrase was a mouthful, he said, wishing aloud for a better name. 'How about black hole?' someone shouted from the audience. That was it. 'I had been searching for just the right term for months, mulling it over in bed, in the bathtub, in my car, wherever I had quiet moments', he later said. 'Suddenly this name seemed exactly right.' He kept using the term, in lectures and on papers and it stuck. (MacPherson 2008) 

besoins du traducteur, nous nous sommes tournés vers une analyse qualitative portant sur deux corpus de taille restreinte. S. Vandaele considère d'ailleurs que les corpus confèrent plusieurs avantages au traducteur pour repérer les métaphores conceptuelles :

Nous proposons, comme moyen d'étude, l'annotation manuelle de corpus en format XML, ce qui permet l'extraction des données en vue d'analyses qualitatives ou quantitatives, mais ce qui fournit également un outil d'apprentissage et de consultation. Grâce à cette approche, il devient possible d'explorer différents aspects nécessaires à la description fine des modes de conceptualisation des langues de spécialité. (Vandaele $2006: 288$ )

Nous avons décidé de constituer un corpus en anglais et un corpus en français à partir d'une idée proposée par Ailish Maher, Stephen Waller et Mary Ellen Kerans (2008) et qui semble apporter l'aide nécessaire au traducteur pour repérer et analyser les métaphores conceptuelles de n'importe quel texte. Le but premier de leur méthode, qui s'adresse principalement aux traducteurs novices, est de constituer un corpus monolingue dans la langue cible et d'utiliser un concordancier pour interroger celui-ci afin de répondre à des questions précises de traduction en langue de spécialité. Cette méthode leur permet de pallier la pauvreté de leurs connaissances linguistiques et spécialisées dans le domaine qu'ils traduisent. En effet, ces traducteurs n'ont pas toujours l'occasion de suivre des formations pour se spécialiser, et cette méthode leur permet d'avoir un accès direct à l'ensemble de la langue de spécialité du domaine concerné et de se former de manière autonome :

For translators working in fields in which they lack linguistic confidence, it can be difficult to acquire specialist knowledge other than by learning on the job or going back to college [...]. A viable alternative is the corpus-guided approach, which consists of systematically collecting target-language texts in the same genre and knowledge area as the source text in order to create a corpus that can be mined using one or several of the software tools available for text analysis. (Maher, Waller \& Kerans $2008: 56$ ) 

comprenant onze textes en anglais et l'autre, onze textes en français. À partir du texte à traduire Goldilocks black holes rédigé par Jenny E. Greene dans Scientific American, nous avons colligé dix articles supplémentaires en anglais et appartenant au genre de la vulgarisation scientifique afin de repérer si les modes de conceptualisation présents dans ce texte se retrouvaient dans d'autres textes rédigés dans la même langue. Ces dix autres textes sont issus des sites web de la NASA, de National Geographic, de l'Université du Michigan et du magazine New Scientist. À l'aide du concordancier AntConc, nous avons repéré de nombreux indices de conceptualisation qui nous ont permis d'inférer la conceptualisation métaphorique gouvernant le concept de trou noir (voir tableau 1).

Nous avons procédé de la même manière pour constituer le deuxième corpus. Nous avons rassemblé onze textes de vulgarisation en français qui nous ont permis de repérer de nombreux indices de conceptualisation et d'inférer la conceptualisation métaphorique en langue cible. Ces articles sont issus des magazines Science et Vie, Sciences et Avenir, Pour la Science ${ }^{4}$, du site web de l'Institut national des sciences de l'univers et du Centre national de la recherche scientifique. Le concordancier AntConc nous a permis de repérer les indices de conceptualisation et d'inférer les modes de conceptualisation qui en résultent. Nous avons utilisé les fonctions «Concordance » et " $N$-grams » du concordancier et lancé une recherche avec les termes «black hole » et " trou noir ». Le concordancier a établi une liste des autres termes les plus souvent directement associés à "black hole» et "trou noir » ou présents dans leur contexte proche. Nous avons ensuite extrait plusieurs exemples de cette liste, avant de les analyser.

Les avantages de cette méthode sont multiples. D'abord, elle permet de gagner un temps considérable par rapport aux recherches que l'étudiant-traducteur aurait dû effectuer pour acquérir les connaissances linguistiques liées au domaine. Ensuite, le traducteur novice est obligé de vérifier ses sources avec un œil très critique pour sélectionner les textes qui constitueront son corpus. Ainsi, il ne peut plus se perdre dans le flot d'informations que propose internet, ni de les accepter sans les remettre en question. En outre, l'utilisation d'un corpus comparable et d'un concordancier permet d'analyser la langue de spécialité sur le plan phraséologique et non plus uniquement sur le plan lexical, comme le font la plupart des dictionnaires. Enfin, elle assure un repérage et une analyse rapides des indices de conceptualisation en langue source et en langue cible afin d'en extraire les modes de conceptualisation et ainsi d'en prendre la meilleure décision traductionnelle. De plus, ces indices de conceptualisation et, par conséquent, ces modes de conceptualisation sont étudiés sur le plan synchronique grâce à cette méthode, tandis que la terminologie retrouvée dans certaines ressources terminologiques semble parfois intégrer des données diachroniques.

\section{La conceptualisation métaphorique de «black hole »}

Partant des données de S. Vandaele en sciences médicales et de $\mathrm{C}$. Resche en économie, nous avons voulu étudier les modes de conceptualisation métaphorique dans le domaine de l'astrophysique. Nous avons fondé notre étude sur des textes de vulgarisation $^{5}$ en anglais et en français pour comprendre quel(s) mode(s) de conceptualisation était (étaient) le(s) plus présent(s) dans ces deux langues. À partir des exemples extraits des corpus comparables, nous remarquons que l'objet céleste étudié 
est représenté à la fois comme un réservoir, un piège ou un aspirateur et comme une personne ou un animal. Nous avons classé quelques exemples dans le tableau 1 où les indices de conceptualisation, c'est-à-dire les unités lexicales témoignant des modes de conceptualisation, sont soulignés.

Tableau 1

\begin{tabular}{|c|c|}
\hline Métaphores du contenant & Métaphores de la personnification \\
\hline $\begin{array}{l}\text { 1.1) Black holes have a reputation of mighty } \\
\text { vacuum cleaners. (Greene } 2012: 42 \text { ) }\end{array}$ & $\begin{array}{l}\text { 1.2) How did these behemoths grow so big, so } \\
\text { fast? (Greene } 2012: 42 \text { ) }\end{array}$ \\
\hline $\begin{array}{l}\text { 2.1) Physicists calculate that a black hole } \\
\text { sucking in surrounding matter at its maximal } \\
\text { rate }[. . .](\text { Greene } 2012: 42)\end{array}$ & $\begin{array}{l}\text { 2.2) We focus on 'active black holes' - ones that } \\
\text { happen to be eating stuff. (Greene } 2012: 43 \text { ) }\end{array}$ \\
\hline $\begin{array}{l}\text { 3.1) A black hole is anything but empty space. } \\
\text { Rather, it is a great amount of matter packed } \\
\text { into a very small area - think of a star ten times } \\
\text { more massive than the Sun squeezed into a } \\
\text { sphere approximately the diameter of New York } \\
\text { City (NASA 2013) }\end{array}$ & $\begin{array}{l}\text { 3.2) "Black holes would have grown only by } \\
\text { more happenstance meals of gas at the galaxy } \\
\text { center - snacks that are not connected with } \\
\text { events shaping the overall evolution of the } \\
\text { galaxy. (Greene } 2012: 47 \text { ) }\end{array}$ \\
\hline $\begin{array}{l}\text { 4.1) Changes in the X-ray spectrum observed by } \\
\text { RXTE during each beat reveal that the } \\
\text { innermost region of the disk emits enough } \\
\text { radiation to push back the gas, creating a strong } \\
\text { outward wind that stops the inward flow [...] } \\
\text { (Reddy 2011) }\end{array}$ & $\begin{array}{l}\text { 4.2) NASA's RXTE Detects 'Hearbeat' of Smallest } \\
\text { Black Hole Candidate. (Reddy 2011) }\end{array}$ \\
\hline $\begin{array}{l}\text { 5.1) Now, with a second system exhibiting } \\
\text { similar types of variability, we really can begin } \\
\text { to test how well we understand what happens at } \\
\text { the brink of a black hole. (Reddy 2011) }\end{array}$ & $\begin{array}{l}\text { 5.2) A mammoth black hole has been discovered } \\
\text { fleeing its host galaxy at high speed, according to } \\
\text { a controversial new study. (Shiga 2008) }\end{array}$ \\
\hline $\begin{array}{l}\text { 6.1) Astronomers spot them when another star } \\
\text { draws near enough for some of the matter } \\
\text { surrounding it to be snared by the black hole's } \\
\text { gravity, churning out x-rays in the process. } \\
\text { (NASA 2013) }\end{array}$ & $\begin{array}{l}\text { 6.2) Astronomers soon realized the source, } \\
\text { known as Swift J1644+57, was the result of a truly } \\
\text { extraordinary event - the awakening of a distant } \\
\text { galaxy's dormant black hole as it shredded and } \\
\text { consumed a star. (Reddy 2011) }\end{array}$ \\
\hline $\begin{array}{l}\text { 7.1) So if the black hole sucks in information } \\
\text { with its intense gravitational pull, then later } \\
\text { disappears entirely, information and all, how } \\
\text { can the laws of quantum physics be preserved? } \\
\text { (Michigan State University 2014) }\end{array}$ & $\begin{array}{l}\text { 7.2) Eventually, however, it will fall into the jaws } \\
\text { of the bigger hole, fattening it up even more. } \\
\text { (Muir 2003) }\end{array}$ \\
\hline \multicolumn{2}{|c|}{$\begin{array}{l}\text { 8) Black holes feed off surrounding accretion disks of material. When they run out of this fuel, they } \\
\text { go dormant. (NASA 2013) }\end{array}$} \\
\hline
\end{tabular}


constatons non seulement que les deux métaphores sont réparties de manière plus ou moins équitable dans le corpus en anglais, mais qu'elles peuvent aussi se chevaucher au sein d'une même phrase, comme dans l'exemple 8, où le trou noir est à la fois un réservoir et une personne qui se nourrit et s'endort.

En conservant les conventions d'écriture de G. Lakoff et M. Johnson (1986 [1980]), selon lesquelles les métaphores conceptuelles sont écrites en petites majuscules (LE DOMAINE CIBLE EST LE DOMAINE SOURCE), nous obtenons les métaphores suivantes pour le terme « black hole» :

a black hole is a vacuum cleaner, a trap or a tank

a black hole is a person, a monster or an animal

Les métaphores relevées dans le corpus nous mènent à la métaphore conceptuelle générale suivante: A BLACK HOLE IS A CONTAINER, étant donné que, d'une part, un aspirateur (ou un réservoir ou encore un piège) est un contenant qui aspire (ou accumule) un contenu et qu'il peut être vidé et que, d'autre part, une personne ou un animal a un corps qui est aussi un contenant qui absorbe et rejette diverses substances (autrement dit du contenu). Cela signifie que même si ces deux modes de conceptualisation sont différents, ils sont toutefois compatibles, car gouvernés par la même métaphore conceptuelle. G. Lakoff et $\mathrm{M}$. Johnson appellent ce type de métaphores des «métaphores ontologiques » (1986 [1980] : 35). Selon les deux auteurs, les métaphores ontologiques nous permettent de concevoir des idées, des émotions, des phénomènes en termes d'objets ou de substances (notamment pour les métaphores du contenant) ou de représenter un concept par une personne ; c'est le cas des métaphores de la personnification (1986 [1980] : 35).

D'après G. Lakoff et M. Johnson, le recours à un mode de conceptualisation plutôt qu'un autre permet de faire ressortir certaines propriétés spécifiques d'un concept :

Le réseau systématique d'expressions métaphoriques qui nous permet de comprendre un aspect d'un concept en termes d'un autre [...] masquera nécessairement d'autres aspects de ce même concept. (1986 [1980] : 20)

Par exemple, l'auteur d'un article sur les trous noirs qui décide (consciemment ou inconsciemment) de recourir à la métaphore de l'aspirateur mettra en avant certaines propriétés du trou noir (qu'il aspire, à l'instar d'une machine) et masquera d'autres propriétés de ce concept. En revanche, le mode de conceptualisation de la personnification peut être sollicité par l'auteur pour mettre en avant les propriétés du trou noir qui font penser à un comportement humain (que le trou noir se réveille, qu'il s'endort, qu'il a des compagnes, etc.) et masquer celles qui font penser à une machine. Le recours à l'un ou l'autre mode de conceptualisation permet de mettre en évidence un aspect particulier du concept de "black hole", même si, in fine, les modes de conceptualisation sont structurés par une métaphore unique.

\section{La conceptualisation métaphorique du trou noir}

41 Maintenant que nous avons cerné la métaphore conceptuelle qui gouverne le terme " black hole » en anglais, nous nous demandons si cette même projection s'opère dans la langue cible, à savoir le français. À partir de notre corpus français, nous avons étudié la conceptualisation métaphorique du trou noir en français. Dans un même temps, nous essayons aussi de comprendre comment le traducteur peut intégrer cette métaphore 
conceptuelle dans sa traduction car, lorsqu'il a repéré la métaphore conceptuelle dans la langue source et la langue cible, le traducteur peut se trouver dans plusieurs cas de figure: si la même métaphore conceptuelle existe dans la langue cible, il doit la transposer. Si la métaphore conceptuelle n'est pas exactement la même, il peut aussi adapter la conceptualisation de la langue source pour qu'elle soit comprise dans la langue cible. Enfin, lorsqu'aucune métaphore conceptuelle adéquate n'existe dans la langue cible, la dernière possibilité consiste à innover et à créer une nouvelle métaphore conceptuelle qui imite l'effet véhiculé dans le texte source (Vandaele 2006 : 287).

Pour appréhender la métaphore conceptuelle qui gouverne les trous noirs en langue française, nous avons interrogé notre corpus en français en utilisant à nouveau les fonctions «N-Grams» et "Concordance» du concordancier AntConc. Nous avons répertorié quelques exemples dans le tableau 2 en soulignant les indices de conceptualisation.

Tableau 2

\begin{tabular}{|c|c|}
\hline Métaphores du contenant & Métaphores de la personnification \\
\hline \multicolumn{2}{|c|}{ 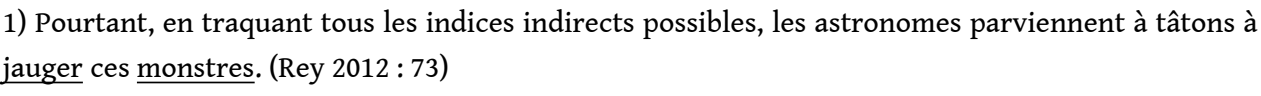 } \\
\hline \multicolumn{2}{|c|}{$\begin{array}{l}\text { 2) Il s'agit d'un trou noir qui vampirise littéralement sa compagne en aspirant une partie de sa } \\
\text { matière. (Rey } 2012: 74 \text { ) }\end{array}$} \\
\hline \multicolumn{2}{|c|}{$\begin{array}{l}\text { 3) Comme les espèces vivantes en symbiose, une galaxie et son trou noir mènent une existence } \\
\text { intimement liée. La galaxie et son environnement } \underline{\text { alimentent le trou noir en gaz. (Ferrari }} \\
\text { \&Tasse 2012) }\end{array}$} \\
\hline $\begin{array}{l}\text { 4.1) À la fois étape ultime de l'effondrement d'un } \\
\text { objet céleste massif et moteurs très puissants } \\
\text { d'accrétion de matière, les trous noirs jouent un rôle } \\
\text { majeur en astrophysique et en cosmologie [...]. } \\
\text { (Lavalle 2012) }\end{array}$ & $\begin{array}{l}\text { 4.2) le célèbre physicien explique que ces } \\
\text { monstres cosmiques sont incompatibles avec } \\
\text { la physique quantique. (Khalatbari 2014) }\end{array}$ \\
\hline $\begin{array}{l}\text { 5.1) Les trous noirs massifs observés par les } \\
\text { astronomes, qui n'ont rien de quantique, seraient } \\
\text { équipés de murs de feu. Anéanti dans un tel mur, } \\
\text { l'observateur qui aurait le malheur de tomber dans } \\
\text { un trou noir serait aussi débarrassé de l'excès } \\
\text { d'information. (Lasota-Hirszowicz 2014) }\end{array}$ & $\begin{array}{l}\text { 5.2) Les trous noirs font aujourd'hui partie } \\
\text { du bestiaire astrophysique classique. } \\
\text { (Lavalle 2012) }\end{array}$ \\
\hline $\begin{array}{l}\text { 6.1) Des observateurs peuvent toujours traverser } \\
\text { tranquillement la surface d'un trou noir massif sans } \\
\text { ressentir quoi que ce soit de particulier. (Lasota- } \\
\text { Hirszowicz 2014) }\end{array}$ & $\begin{array}{l}\text { 6.2) Qu'advient-il de la matière lorsqu'elle } \\
\text { est engloutie par un trou noir? } \\
\text { (Riazuelo } 2014: 114 \text { ) }\end{array}$ \\
\hline
\end{tabular}




\begin{tabular}{|l|l|}
\hline $\begin{array}{l}\text { 7.1) Comment les trous noirs supermassifs peuvent- } \\
\text { ils capturer de la matière sans émettre beaucoup de } \\
\text { rayonnement? (Grosso \& Porquet 2013) }\end{array}$ & $\begin{array}{l}\text { 7.2) L'accrétion de matière par des trous } \\
\text { noirs gigantesques est sans doute } \\
\text { responsable de l'éclat intense des galaxies } \\
\text { dites actives. Ces ogres pourraient avoir } \\
\text { pour origines des trous noirs intermédiaires. } \\
\text { (Lavalle 2012) }\end{array}$ \\
\hline $\begin{array}{l}\text { 8.1) Mais la « taille " d'un trou noir, définie comme } \\
\text { le rayon de son horizon [...] (Cassé 2009:34) }\end{array}$ & $\begin{array}{l}\text { 8.2) Le trou noir supermassif présent au } \\
\text { centre de notre Galaxie a connu un passé } \\
\text { agité ces derniers siècles. (Terrier et alii } \\
2010)\end{array}$ \\
\hline
\end{tabular}

Nous constatons que le corpus français est gouverné par les mêmes modes de conceptualisation que le corpus anglais, qui transparaissent à travers la métaphore de l'aspirateur ou du réservoir et la métaphore du l'homme ou du monstre. De plus, en français également, ces métaphores peuvent se chevaucher tout en restant compatibles, comme en témoignent les exemples 1,2 et 3 du tableau 2 . Enfin, les expressions métaphoriques sont sensiblement similaires dans les deux langues. À l'aide de plusieurs exemples, nous avons établi des parallèles entre les deux tableaux pour montrer cette similitude. Les trous noirs sont considérés comme des machines ayant une force d'aspiration: "sucking in" (exemple 2.1 du tableau 1) et "aspirant» (exemple 2 du tableau 2). Les trous noirs peuvent aussi prendre la forme d'un monstre : "behemoths» (exemple 1.2 du tableau 1) et "monstres cosmiques» (exemple $4.2 \mathrm{du}$ tableau 2). Nous en concluons que ces deux langues conceptualisent la notion de trou noir de la même manière.

44 À partir de ces observations, le traducteur peut donc décider avec assurance de conserver dans sa traduction la même métaphore conceptuelle qu'en langue source et de recourir à des expressions métaphoriques analogues dans sa traduction. Il n'est ni nécessaire d'adapter la métaphore conceptuelle repérée en anglais ni d'en créer de nouvelles. Nous pensons dès lors qu'il semble plus adéquat de traduire «black hole » par «trou noir» et non par «astre occlus ». D'autant plus que la notion d'astre occlus semble être gouvernée par une métaphore conceptuelle en opposition avec la métaphore conceptuelle cernée ci-dessus en anglais et en français, comme nous allons le voir dans la section suivante.

\section{Les problèmes de traduction liés à l'utilisation du terme " astre occlus »}

En lisant la fiche terminologique de Termium Plus (voir section 1), on pourrait penser que le terme "astre occlus » fait référence à la métaphore conceptuelle d'une entité finie et délimitée qui est hermétique. Cette métaphore conceptuelle ne correspond pas à celle du « trou noir » qui évoque une entité infinie, invisible et aspirant toute matière environnante. Cette différence pourrait mener à des problèmes de traduction. Considérons les problèmes suivants :

If black holes are « black », how do scientists know they are there? (Smith 2008) 
ce cas précis, le traducteur n'aurait pas été en mesure de traduire le terme «black hole » par « astre occlus », car cette traduction n'aurait pas pu véhiculer l'image que les trous noirs sont invisibles parce qu'ils sont justement noirs. Voici un deuxième exemple :

Black holes have a reputation of mighty vacuum cleaners. (Greene $2012: 42$ )

Dans ce deuxième exemple, il semble également difficile de traduire "black hole » par «astre occlus ». S'il avait privilégié le terme "astre occlus», le traducteur aurait été contraint d'ajouter une précision telle que «contrairement à ce que l'on pourrait imaginer... » ou encore " même si l'adjectif occlus laisse penser le contraire... » pour justifier cette association quelque peu contradictoire entre un astre hermétique et le fait que ce même astre soit doté d'une grande force d'aspiration.

Enfin, utiliser le terme « astre occlus » pour désigner la notion de black hole reviendrait non seulement à évoquer une métaphore conceptuelle différente qui pourrait, dans certains cas, compliquer quelque peu la tâche du traducteur, mais cela engendrerait aussi une contradiction avec d'autres concepts qui s'inscrivent dans le même cadre métaphorique du trou noir et qui sont gouvernés par les mêmes modes de conceptualisation. C'est notamment le cas du concept opposé du black hole, qui est celui du white hole (trou blanc) dont voici la définition :

A white hole is the theorized time reversal of a black hole. The event horizon of a black hole attracts matter, so the event horizon of a white hole ejects matter. (Coffey 2010)

C'est également le cas de wormhole, que les experts définissent comme suit :

Wormholes are solutions to the Einstein field equations for gravity that act as 'tunnel', connecting points in space-time in such a way that the trip between the points through the wormhole could take much less time than the trip through normal space. The first wormhole-like solutions were found by studying the mathematical solution for black holes. There it was found that the solution lent itself to an extension whose geometric interpretation was that of two copies of the black hole geometry connected by a 'throat'. The throat is a dynamical object attached to the two holes that pinches off extremely quickly into a narrow link between them. (Holman 1997)

Les concepts de trou blanc et de trou de ver sont apparus après celui de trou noir, mais ils semblent être gouvernés par les mêmes modes de conceptualisation que ce dernier. Le fait que ces concepts appartiennent tous au même cadre métaphorique n'est pas anodin: ce sont des métaphores filées qui ont été forgées sur base de la conceptualisation métaphorique du trou noir. Si l'on en croit S. Vandaele, il serait plus facile d'intégrer de nouveaux concepts à un domaine si leur appellation fait transparaître la même métaphore conceptuelle gouvernant un concept déjà intégré au domaine en question :

On peut ainsi faire l'hypothèse qu'un nouveau concept dont la dénomination est motivée par un mode de conceptualisation déjà existant s'intègre beaucoup facilement que s'il met en jeu une conceptualisation nouvelle. (Vandaele 2006 : 287)

51 Si le traducteur décide de conserver le terme « astre occlus » pour traduire la notion de black hole, il se peut que, dans certains cas, il crée une contradiction avec les modes de conceptualisation de white hole et de wormhole. Considérons le problème de traduction suivant :

A white hole is the theorized time reversal of black hole. (Coffey 2010) 
La dénomination « trou blanc » a été directement conçue autour de la même métaphore conceptuelle que celle qui gouverne le trou noir, comme nous l'avons mentionné cidessus. Or, s'il traduit "black hole " par "astre occlus », et "white hole» par " trou blanc » le traducteur va dans une même phrase utiliser des termes qui renvoient à deux métaphores conceptuelles différentes et incompatibles, alors qu'il semblerait plus logique d'utiliser des termes qui sont liés à la même conceptualisation métaphorique.

Le même type de contradiction est observable si le traducteur décide de traduire le concept de "black hole» par "astre occlus» dans un passage sur les trous de ver, puisqu'il serait incapable d'expliquer que deux "astres ", que l'on imagine comme des sphères solides et hermétiques, se rejoignent pour former un tunnel. Ici, la contradiction se situe dans l'utilisation d'une expression métaphorique faisant référence à de la matière alors qu'elle devrait renvoyer à une image de vacuité. Considérons les exemples suivants :

The first wormhole-like solutions were found by studying the mathematical solution for black holes. There it was found that the solution lent itself to an extension whose geometric interpretation was that of two copies of the black hole geometry connected by a 'throat'. The throat is a dynamical object attached to the two holes that pinches off extremely quickly into a narrow link between them. (Holman 1997)

Wormholes contain two mouths, with a throat connecting the two. [...] Certain solutions of general relativity allow for the existence of wormholes where the mouth of each is a black hole. (Redd 2013)

Ces exemples décrivent la manière dont deux trous noirs peuvent se rejoindre pour créer la gorge (ou le tunnel) du trou de ver. Les trous noirs situés de part et d'autre de la gorge constituent les bouches du trou de ver. Il aurait été difficile de véhiculer cette image en utilisant le terme "astre occlus ", étant donné que deux sphères solides ne peuvent pas se rejoindre pour former un tunnel entre elles, et qu'elles ne font pas penser à des ouvertures, qui sont conceptualisées par des bouches.

\section{Conclusion}

5 En résumé, nous avons voulu illustrer le fait que les bases de données, les thésaurus, ou encore les glossaires ne constituaient pas des outils de choix pour repérer et analyser la conceptualisation métaphorique du terme "BLACK HOLE», d'autant plus que les recommandations terminologiques présentes dans ces ressources peuvent entrer en contradiction avec la métaphore conceptuelle propre au domaine des trous noirs.

En revanche, la constitution de deux corpus comparables semble représenter un outil particulièrement utile pour l'étudiant-traducteur; elle lui permet de repérer les indices de conceptualisation, d'en inférer la conceptualisation métaphorique pour la langue source et la langue cible et, ainsi, de prendre la meilleure décision traductionnelle. Après avoir repéré et analysé les indices métaphoriques qui parsèment le domaine des trous noirs en anglais et en français à l'aide d'un concordancier, nous en avons induit les modes de conceptualisation qui leur sont directement liés. Nous en sommes venus à la conclusion que la métaphore conceptuelle des trous noirs est identique en anglais et en français et que la traduction la plus adéquate de «black hole » est « trou noir » et non " astre occlus ». L'analyse qualitative d'un échantillon de textes semble donc être la méthode le plus adaptée pour étudier les métaphores conceptuelles des textes 
scientifiques, par rapport aux dictionnaires, et aux ressources terminologiques, qui sont les outils les plus souvent sollicités par les étudiants-traducteurs lors des cours de traduction spécialisée.

Enfin, étant donné le renouveau rapide de la physique et les divergences entre les écoles de pensée, il serait intéressant de vérifier si l'émergence de nouveaux paradigmes mènera à la naissance de nouvelles conceptualisations métaphoriques et/ ou à l'éventuelle modification des modes conceptuels préexistants via des mutations métaphoriques ou des projections comme l'a constaté C. Resche pour l'anglais économique (2002). Nous avons par exemple constaté à travers nos recherches que le concept de "black hole» tel qu'il est compris en astrophysique (domaine source) a été repris et appliqué, par projection, au domaine de l'informatique (domaine cible) pour désigner l'endroit où aboutit un courriel ou toute autre information qui disparaît mystérieusement du web. Une recherche plus approfondie permettrait certainement de déceler d'autres projections similaires.

\section{BIBLIOGRAPHIE}

Académie française. Non daté. «Le français aujourd'hui ». Consulté le 30 avril 2014. <http:// www.academie-francaise.fr/la-langue-francaise/le-francais-aujourdhui>.

« Black hole ». 2014. Techopedia, consulté le 10 février 2014 <http://www.techopedia.com/ definition/3159/black-hole>.

Boucher, France, Conrad Ouellon \& Jacques Gosselin. 2009. Plan stratégique en matière de politique linguistique. Québec : Bibliothèque et Archives nationales du Québec.

Boyd, Richard. 1993 [1979]. "Metaphor and theory change: What is 'metaphor' a metaphor for?". In Ortony, A. (dir.), Metaphor and Thought. $2^{\mathrm{e}}$ édition. Cambridge : Cambridge University Press, 481-532.

Brown, Theodore. 2003. Making Truth. Metaphor in Science. Urbana and Chicago : University of Illinois Press.

bühler, Hildegun. 1982. « General theory of terminology and translation studies ». Meta: Translators' Journal. Vol 27/4. 425-431.

Coffey, Jerry. 2010. « White Holes ». Universe today, consulté le 17 février $2014<$ http:// www.universetoday. com/76909/white-holes/>.

Gaudin, François. 2003. Socioterminologie, une approche sociolinguistique de la terminologie. Bruxelles : Duculot de Boeck.

Hesse, Mary B. 1966. Models and Analogies in Science. Notre Dame, IN : University of Notre Dame Press.

Holman, Richard. 1997. « Follow-up: What exactly is a 'wormhole'? Have wormholes been proven to exist or are they still theoretical? ». Scientific American, consulté le 11 mars 2014 <http:// www.scientificamerican.com/article/follow-up-what-exactly-is/>. 
Lakoff, George. 1993 [1979]. « The contemporary theory of metaphor ». In Ortony, A. (dir.), Metaphor and Thought. $2^{\mathrm{e}}$ édition. Cambridge : Cambridge University Press, 202-251.

Lakoff, George \& Mark Johnson. 1986 [1980]. Les Métaphores dans la vie quotidienne. Trad. Michel de Fornel (Metaphors We Live By. Chicago : University of Chicago Press). Paris : Les Éditions de Minuit.

Lakoff, George \& Rafael Núñez. 2000. Where Mathematics Comes From. New York : Basic Books.

Laplace, Pierre Simon. 1798. Exposition du système du monde, $2^{\mathrm{e}}$ édition, consulté le 10 février 2014 <http://gallica.bnf.fr/ark:/12148/btv1b8615813r.r=exposition+du+système+du+monde.langFR>.

Lombry, Thierry. 2000. « Le trou noir ». Sur le bord du monde, consulté le 15 février 2014. <http:// polywww.in2p3.fr/ mine/cosmo/Lombry.pdf >.

Luminet, Jean-Pierre. 2011. Illuminations : cosmos et esthétique. Paris : Odile Jacob.

MacPherson, Kitta. 2008. « Leading physicist John Wheeler dies at age 96 ». Current Stories, consulté le 10 février 2014 <http://www.princeton.edu/main/news/archive/S20/82/08G77/ index.xml?section=topstories\&path=/main/news/archive/S20/82/08G77/index.xml\&prev=1>.

Maher, Ailish, Stephen Waller \& Mary Ellen Kerans. 2008. « Acquiring or enhancing a translation specialism: the monolingual corpus-guided approach ». The Journal of Specialised Translation 10, 57-73.

Redd, Nola. 2013. « What is a wormhole?». SPACE, consulté le 30 avril $2014<$ http:// www.space.com/20881-wormholes.html>.

Resche, Catherine. 2002. «La métaphore en langue spécialisée, entre médiation et contradiction: étude d'une mutation métaphorique en anglais économique ». ASp 35-36, 103-119.

Smith, Heather. 2008. «What is a black hole?». NASA Educational Technology Services, consulté le 30 avril 2014 <http://www.nasa.gov/audience/forstudents/k-4/stories/what-is-a-black-holek4.html\#.U2SbutxARM0>.

Temmerman, Rita. 2000. Towards New Ways of Terminology Description: the sociocognitive approach. Amsterdam : John Benjamins.

Vandaele, Sylvie. 2002. « Métaphores conceptuelles en traduction biomédicale et cohérence ». TTR : traduction, terminologie, rédaction 15/1, 223-239.

Vandaele, Sylvie. 2006. «Conceptualisation métaphorique en biomédecine : importance pour le processus traductionnel ». Congrès de l'ABECAN (Associação Brasileira de Estudos Canadenses), Gramado, novembre 2005, 281-294.

Xuan Thuan, Thrinh. 1998. Le chaos et l'harmonie : la fabrication du Réel. Paris : Les Éditions Fayard.

\section{Références des textes du corpus français (cités dans le texte)}

Cassé, Michel. 2008. « Phénoménologie du trou noir chantant ». In Cassé, M., Trous noirs en pleine lumière. Paris : Éditions Odile Jacob, 31-37.

Ferrari, Chiara \& Cyril Tasse. 2012. « Un trou noir supermassif souffle une bulle géante de gaz chaud ». Actualités du CNRS-INSU, consulté le 12 mars 2014 <http://www.insu.cnrs.fr/node/4041>. Grosso, Nicolas \& Delphine Porquet. 2013. « La faible luminosité du trou noir supermassif de notre galaxie enfin expliquée ». Communiqué de presse du CNRS, consulté le 12 mars 2014 <http:// www2.cnrs.fr/presse/communique/3207.htm>.

Khalatbari, Azar. 2014. "Stephen Hawking : 'les trous noirs n'existent pas' ». Sciences et Avenir, consulté le 12 mars 2014 <http://www.sciencesetavenir.fr/fondamental/20140127.OBS3956/ stephen-hawking-les-trous-noirs-n-existent-pas.html>. 
Lasota-Hirszowicz, Jean-Pierre. 2014. « Einstein garantit une traversée paisible de l'horizon d'un trou noir ». Actualités du CNRS-INSU, consulté le 12 mars 2014 <http://www.insu.cnrs.fr/node/ 4769>.

Lavalle, Julien. 2012. « Les trous noirs de masse intermédiaire ». Dossier Pour la Science 75, 102-109. Rey, Benoit. 2012. « Observer les trous noirs ». Science et Vie Hors Série 259, 73-92.

Riazuelo, Alain. 2014. « À l'horizon des trous noirs ». Dossier Pour la Science 83, 114-119.

Terrier, Regis et alii. 2010. « Le passé agite du trou noir de notre Voie lactée ». Communiqué de presse du CNRS, consulté le 12 mars 2014 <http://www2.cnrs.fr/presse/communique/1894.htm>.

Références des textes du corpus anglais (cités dans le texte)

« Black holes ». 2013. NASA, consulté le 12 mars 2014. <http://science.nasa.gov/astrophysics/ focus-areas/black-holes/>.

Greene, Jenny. 2012. « Goldilocks Black Holes ». Scientific American 306, 40-47.

Muir, Hazel. 2003. « Second black hole may lurk at Milky Way's heart ». New Scientist, consulté le 12 mars 2014 <http://www.newscientist.com/article/dn3847-second-black-hole-may-lurk-atmilky-ways-heart.html\#.U1E4tdxARM1>.

« Plugging the hole in Hawking's black hole theory ». 2014. Michigan State University. Science news line, consulté le 12 mars 2014 <http://www.sciencenewsline.com/summary/

2014032423370024.html>.

Reddy, Francis. 2011. « NASA's RXTE detects 'heartbeat' of smallest black hole candidate ». NASA, consulté le 12 mars 2014 <http://www.nasa.gov/topics/universe/features/black-hole-

heartbeat.html>.

Shiga, David. 2008. « Monster black hole escaping home galaxy ». New Scientist, consulté le 12 mars 2014. <http://www.newscientist.com/article/dn13803-monster-black-hole-found-escapinghome-galaxy.html\#.U1E15dxARM0>.

\section{NOTES}

1. <http://www.planete-astronomie.com/lexique-d-astronomie-de-planete-astronomie.html>

2. <http://www.espace-sciences.org/planetarium/glossaire-astronomie>

3. <http://www.cndp.fr/thesaurus-motbis/site/>

4. Dans le cas de ce magazine, nous nous sommes assuré que les articles étaient rédigés par des auteurs francophones. Ce ne sont pas des traductions.

5. Il est à noter que des résultats probablement différents auraient été observés si nous avions étudié le concept de trou noir à partir de textes relevant d'un niveau d'expert. Cependant, étant donné que notre étude se fonde sur des observations faites chez les étudiants-traducteurs, nous avons décidé de concentrer notre étude sur les textes de vulgarisation portant sur les trous noirs. 


\section{RÉSUMÉS}

Dans cet article, l'auteur analyse les modes de conceptualisation dans des textes de vulgarisation scientifique sur les trous noirs. Elle constate que le traducteur novice a souvent pour premier réflexe de se tourner vers les bases de données pour résoudre des problèmes ponctuels de traduction en langue de spécialité. Elle argumente que ces bases de données ne constituent pas la solution optimale pour traduire un texte dans lequel les métaphores conceptuelles sont omniprésentes. D'après ses observations, la constitution de corpus constitue une démarche intéressante pour, d'une part, repérer la conceptualisation métaphorique qui gouverne un concept et, d'autre part, prendre les meilleures décisions pour traduire les expressions métaphoriques qui y sont liées. Au moyen d'une analyse contrastive anglais-français, l'auteur montre que l'accès aux modes de conceptualisation est déterminant pour l'étudiant-traducteur étant donné que ces derniers lui fournissent un indice probant sur la terminologie à adopter dans sa traduction.

In this article, the author analyzes the metaphorical conceptualization in popular science articles about black holes. She has noticed that translation students tend to first browse databases in order to solve isolated translation problems in LSP. She argues that these databases are not ideal tools to translate a text that is filled with conceptual metaphors. Compiling a corpus of a few texts appears to provide adequate tools to translation students in order to identify the metaphorical conceptualization that lies beneath a concept and to make the best decisions to translate the metaphorical expressions directly linked to the conceptualization. She attempts to give a contrastive analysis in English and French in order to show that grasping the conceptualization modes is crucial for translation students, for they give strong indications about the terminology to use when translating.

\section{INDEX}

Keywords : astrophysics terminology, metaphorical projection, qualitative corpus analysis, specialized translation, terminological database

Mots-clés : analyse qualitative de corpus, base de données terminologiques, projection métaphorique, terminologie de l'astrophysique, traduction spécialisée

\section{AUTEUR}

\section{CHARLÈNE MEYERS}

Charlène Meyers est traductrice et doctorante à l'Université de Mons (FTI-EII) en Belgique, où elle a obtenu un Master en traduction en 2012 et un Master complémentaire en linguistique appliquée en 2013. Ses langues de travail sont le français, l'anglais, le danois, le norvégien et le suédois. Elle travaille actuellement en tant que doctorante et assistante dans le service de traduction spécialisée et de terminologie, sous la direction de Christine Michaux. Sa thèse en cours porte sur une étude contrastive en anglais et en français des métaphores conceptuelles dans le domaine de l'astrophysique. Ses principaux domaines de recherche sont la linguistique cognitive, la traduction spécialisée et la terminologie. charlene.meyers@umons.ac.be 